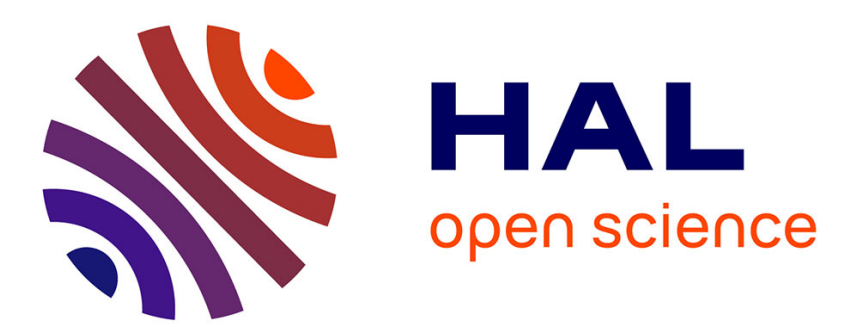

\title{
Using DL_POLY to study the sensitivity of liquid structure to potential parameters
}

\author{
Ruth Lynden-Bell, Tristan Youngs
}

\section{To cite this version:}

Ruth Lynden-Bell, Tristan Youngs. Using DL_POLY to study the sensitivity of liquid structure to potential parameters. Molecular Simulation, 2007, 32 (12-13), pp.1025-1033. 10.1080/08927020600823133 . hal-00514983

\section{HAL Id: hal-00514983 \\ https://hal.science/hal-00514983}

Submitted on 4 Sep 2010

HAL is a multi-disciplinary open access archive for the deposit and dissemination of scientific research documents, whether they are published or not. The documents may come from teaching and research institutions in France or abroad, or from public or private research centers.
L'archive ouverte pluridisciplinaire HAL, est destinée au dépôt et à la diffusion de documents scientifiques de niveau recherche, publiés ou non, émanant des établissements d'enseignement et de recherche français ou étrangers, des laboratoires publics ou privés. 


\section{Molecular Simulation \\ Journal of \\ Experimental Nanoscience \\ $\because$ Taylor \& Francis \\ Taglor S Francis Group}

\section{Using DL_POLY to study the sensitivity of liquid structure to potential parameters}

\begin{tabular}{|c|c|}
\hline Journal: & Molecular Simulation/Journal of Experimental Nanoscience \\
\hline Manuscript ID: & GMOS-2006-0062.R1 \\
\hline Journal: & Molecular Simulation \\
\hline $\begin{array}{r}\text { Date Submitted by the } \\
\text { Author: }\end{array}$ & 22-May-2006 \\
\hline Complete List of Authors: & $\begin{array}{l}\text { Lynden-Bell, Ruth; Cambridge University, Chemistry } \\
\text { Youngs, Tristan; Atomistic Simulation Centre, Queen's University } \\
\text { Belfast, School of Maths and Physics }\end{array}$ \\
\hline Keywords: & $\begin{array}{l}\text { Molecular dynamics, Hydrogen bonding, Force fields, Liquid } \\
\text { structure }\end{array}$ \\
\hline
\end{tabular}




\title{
Using DL_POLY to study the sensitivity of liquid structure to potential parameters
}

\author{
R. M. Lynden-Bell ${ }^{a},{ }^{b}$ and T. G. A. Youngs ${ }^{b}$ \\ ${ }^{a}$ University Chemical Laboratory, Lensfield Road, \\ Cambridge CB2 1EW, UK. \\ ${ }^{b}$ Atomistic Simulation Centre, School of Mathematics and Physics, \\ Queen's University, Belfast BT7 1NN, UK.
}

(Received 00 Month 200x; In final form 00 Month 200x)

\begin{abstract}
Two case studies are presented showing the local structure in liquids and how it responds to changes in the intermolecular potential. The idea is to use realistic and unrealistic potentials in order to determine the sensitivity of local liquid structure to potential parameters. The first case study concerns two families of modified water models. In the 'hybrid' family the hydrogen bond strength is reduced, but the geometry kept constant; in the second family the molecular geometry is changed by reducing the bond angle, keeping a constant molecular dipole moment. The local structure is measured by radial distribution functions, three dimensional probability distribution functions and three body angular correlations. The second case study concerns the ionic liquid dimethylimidazolium chloride $\left(\left[\mathrm{C}_{1}\right.\right.$ mim $\left.] \mathrm{Cl}\right)$. The effect of reducing the hydrogen bonding potential of the cations while maintaining their charge is examined.
\end{abstract}

\section{Introduction}

In many ways the liquid state of matter is the most difficult to understand experimentally and theoretically. Unlike the gaseous state molecules are nearby and interact; unlike the solid state there is no long range order. Since the pioneering work of people such as Metropolis, Rahman Alder and Wainwright, Harp and Berne $(1 ; 2 ; 3)$, computer simulation has proved to be a good way of studying liquids on the molecular scale. One technique used for such studies is the method of classical molecular dynamics (4). In this method Newtonian equations of motion are solved for the motion of a large number of molecules contained in a cell with periodic boundaries. Although the principles are straightforward and it is feasible to write one's own computer program, there are many advantages in using well tested and optimised code. We have found the DL_POLY program (5) and its subroutines are both easy to use and easy to adapt for particular problems, and most of RMLB's work since 1995 has been performed with this program. To perform classical molecular dynamics simulations one needs to supply a prescription for the intermolecular potential of sets of molecules at varying separations and orientations. For molecular liquids two-body site-site interactions are commonly used and give at least a qualitative picture of liquid structure and dynamics on a molecular scale.

Liquids have no long-range structure, but often show significant local structure. One good example is water, which tends to have a tetrahedral arrangement of neighbours with four to five close neighbours, and another example is a molten salt where cations tend to be surrounded by anions and vice-versa. In this paper we use water and the room-temperature molten salt dimethylimidazolium chloride $\left(\left[\mathrm{C}_{1} \mathrm{mim}\right] \mathrm{Cl}\right)$ as case studies for investigations into the sensitivity of the local liquid structure to the form of the intermolecular potential.

Corresponding author E-mail: rmlb@cam.ac.uk

E-mail: t.youngs@qub.ac.uk

Molecular Simulation

ISSN 0892-7022 print/ISSN 1029-0435 online (c) 200x Taylor \& Francis

http://www.tandf.co.uk/journals

DOI: $10.1080 / 0892702$ YYxxxxxxxx

http://mc.manuscriptcentral.com/tandf/jenmol 
The details of all liquid properties depend on the intermolecular potential, which is, in principle, completely determined by the equations of quantum mechanics applied to electrons and nuclei. However, approximations must be made. In classical molecular dynamics calculations the nuclei are treated as classical particles and the intra and intermolecular potentials approximated by some function which must be specified. Much effort has been expended in finding approximate functions which combine ease of computation with a reasonably accurate description of the liquid. But classical molecular dynamics calculations can also be used in a different way - namely to investigate which properties depend on what aspects of the intermolecular potential. We can alter the potential in a controlled way, and find the resulting changes in properties such as local structure, solute chemical potential and dynamics (to name but a few examples). We can then see whether the solvation properties correlate with changes in local structure or whether the changes in dynamics correlate with changes in thermodynamics.

The representation of the average local structure in a molecular liquid is not easy. Even if we confine ourselves to two-molecule correlations one needs one distance and three angles to described the position and orientation of a water molecule in a set of molecular axes placed in a reference water molecule. The simplest representation is a site-site radial distribution function $g_{i j}(r) . g_{i j}(r)$ is the ratio of the probability of finding a site of type $j$ at distance $r$ from a site of type $i$ to the probability of finding it at the same distance from a random point. This gives no directional information, but is simple to display in a one dimensional plot. Another representation, the three dimensional probability distribution function, shows the probability of finding a site of type $j$ at position $\mathbf{r}$ in local axes fixed on the molecule containing site $i$. These functions can be visualised by showing contour surfaces in three dimensions or by two-dimensional cuts through the distribution. In this paper we show 3 -dimensional contour surfaces. These two representations depend only on two-molecule correlations. It is possible to represent aspects of three body correlations, but such representations tend to be specific to the problem. For example in water we may define a measure of the tetrahedrality of the local environment by means of a tetrahedrality parameter or we may plot the distribution of angles (or their cosines) subtended by pairs of nearby oxygen sites.

We present two case studies of the way in which the local structure of the liquid depends on potential parameters. In the first we look at aspects of families of liquids formed from modifying a common water potential. Liquid water is common on the planet earth and is essential for life as we know it. It has many properties which are unusual when compared to other substances which are liquid at ambient conditions. The latent heat of vaporisation is high showing that there is a strong attraction between molecules. The liquid is denser than the solid at the triple point and has a negative coefficient of thermal expansion at low temperatures. It has a high dielectric constant and is a selective solvent for polar solutes and barely dissolves oily materials. It does not wet waxes. On the molecular scale the molecular volume is unusually small (only about $30 \mathrm{~A}^{3}$ under ambient conditions). These properties depend directly or indirectly on the strong hydrogen bonds formed by each molecule, which can form two bonds by donation and can accept hydrogen bonds. In ice I $h$ each molecule forms and accepts two hydrogen bonds in a local tetrahedral arrangement which persists locally in liquid water.

We have studied local structure in two families of modified water models. In the first family (bent models) we decrease the bond angle from tetrahedral (6), keeping constant the molecular dipole and density at ambient conditions. In the second family (hybrid models) we keep the geometry constant but change the relative strengths of the Lennard-Jones and electrostatic parts of the intermolecular potential (7). This has the effect of reducing the hydrogen bond strength and making the liquid more like a Lennard-Jones fluid a model for a normal organic solvent. Again the density and molecular dipole moment are kept constant.

This case study is part of a continuing investigation aimed at trying to understand the origin of the unique properties of water. The bent family is one in which strong hydrogen bonds are retained but the geometry is changed. This alters the local network structure in the resulting liquid. The hybrid family, on the other hand, is one in which only the hydrogen bond strength is reduced. The question that is addressed in this paper is how the local liquid structure changes as a result of these counter-factual potential alterations. In earlier work (7) we considered the effects of these changes on some of the anomalous properties of water, 
while work under way is aimed at investigating the changes in hydrophobic and hydrophilic solvation.

In the second case study we modify aspects of an ionic liquid forcefield. Ionic liquids are fluids composed entirely of ions and are liquid at ambient temperatures, exhibit negligible vapour pressures, and have proven able to dissolve both organic and inorganic material allowing same-phase reactions involving exotic combinations of reactants. The resulting biological, industrial and environmental significance has put this family of compounds firmly at the centre of interest of many research groups. While the sheer number of different ionic liquids available provides the researched with a healthy selection of new solvents to choose from, on the other hand experimental characterisations are also spread over this number. The end result of this is that, while applications of ionic liquids are studied freely, the fundamental properties which underly the effects are less well-known owing to the scarcity of experimental characterisation.

In this context, classical simulation is becoming increasingly more common, in order that these systems may be understood more clearly on the molecular level. It should be recognised, however, that the emerging area of IL simulation and the relative novelty of these systems means that organic molten salts are not catered for in established forcefields. In order to address this a number of different forcefields have been proposed and validated, commonly as the first step in any group's foray into the field (8). Predominantly these are composed from existing general parameter sources such as OPLS, CHARMM or AMBER, with $a b$ initio-derived values inserted where no suitable data are available. The success of new formulations is usually judged through reproduction of measured liquid densities since very little other experimental data exists. Most of the detailed structural data available $(9 ; 10 ; 11 ; 12)$ concentrates on one of the simplest imidazolium-based ionic liquids available - $\left[\mathrm{C}_{1} \mathrm{mim}\right] \mathrm{Cl}$. From the point of view of forcefield development, this is perfectly acceptable since it provides ample data on which to generate and optimise 'model' potentials, understand in detail the features and dynamics of processes occurring at the molecular level, and then apply this knowledge to more complex ionic liquid systems. Through theoretical investigations such as those described here, we may alter the interactions in the system in ways that are simply not possible in real-world experiments, and which allow for the examination of effects (or lack thereof) arising from tailoring individual elements describing the character of the forcefield.

One issue surrounding ionic liquid systems is the degree of hydrogen bonding existing between cations and anions. For common imidazolium-based ionic liquids the only available hydrogen bonding donors are $\mathrm{C}-\mathrm{H}$ groups, which can make hydrogen bonds as they are part of a charged ring system. Direct evidence for hydrogen bonding in the imidazolium halide family of ionic liquids exists, while for poorly coordinating anions such as tetrafluoroborate and hexafluorophosphate such interactions have been proposed (see the studies of Heimer et al. (13) and Berg et al. (14) and references therein). Recent ab initio molecular dynamics studies on $\left[\mathrm{C}_{1} \mathrm{mim}\right] \mathrm{Cl}$ have also confirmed the existence of $\mathrm{C}-\mathrm{H} \cdots \mathrm{Cl}$ hydrogen bonds $(10 ; 11 ; 12)$. Here we investigate the immediate effect of the strength of the hydrogen bonding potential of the ring $\mathrm{C}-\mathrm{H}$ groups on the structure of the ionic liquid $\left[\mathrm{C}_{1} \mathrm{mim}\right] \mathrm{Cl}$. In this ionic liquid the local electrostatic field around the cation depends on the distribution of charges on the atomic sites (15). At long distances the total charge of +1 is the only important term, but at short distances both the details of the charge distribution and short range repulsive forces affect the local interactions. The extent of hydrogen bonding, for example, depends on the charges on the ring $\mathrm{C}-\mathrm{H}$ groups. In the same spirit as the first case study, we change the hydrogen bonding potential of the cation by gradual reversal of the local $\mathrm{C}-\mathrm{H}$ dipoles of the imidazolium ring and examine the resulting structure. The question that is addressed is the importance of hydrogen-bonding as opposed to molecular shape in determining the local structure of the liquid.

It is important to realise that we do not claim to get improved intermolecular force fields in these studies, rather we are trying to determine the extent to which local structure in liquids is sensitive to potential parameters. This work complements other investigations which will examine how solvation and anomalous behaviour in water and related liquids depend on the strength of hydrogen-bonding in these systems and on the local liquid network which results from molecular geometry. 


\section{Technical details}

\section{$2.1 \quad$ Intermolecular potentials}

2.1.1 Modified water models. The intermolecular potentials of the two families of model liquids are based on the SPC/E model (16). In this model the interaction consists of a Lennard-Jones centre on the oxygen atom and charges on each atom site. The molecules are rigid. In the hybrid family the epsilon of the Lennard Jones interaction is increased, which has the effect of reducing the hydrogen-bond strength and changing the liquid from one that is tetrahedral to one that is more like a Lennard-Jones fluid (7). In the bent family the bond angle is changed, keeping the charges and molecular dipole moments the same (6). Details of the intermolecular potentials are given in table 1. Liquid properties are given in table 2 including the relative hydrogen bond strength in dimers of molecules of the hybrid families.

2.1.2 The ionic liquid $\left[\boldsymbol{C}_{\mathbf{1}} \mathrm{mim}\right] \mathrm{Cl}$. For $\left[\mathrm{C}_{1} \mathrm{mim}\right] \mathrm{Cl}$, the starting model corresponds to that generated from a force matching optimisation based on ab initio data (17) and is non-polarisable and fully flexible. To generate different electrostatic models, the charges of the $\mathrm{C}$ and $\mathrm{H}$ atoms were taken and adjusted by scalar additions of $+\Delta q$ and $-\Delta q$ respectively, where $\Delta q=\delta\left(q_{\mathrm{H}}-q_{\mathrm{C}}\right)$ and $\delta$ is the degree of polarisation we impose on the bond which ranges from 0.0 (original charges) to 1.0 ('reversed dipole') in steps of 0.2.

\section{$2.2 \quad$ Simulation details}

Runs for the modified water models were carried out at $298 \mathrm{~K}$ and $\mathrm{p}=0.001 \mathrm{kBar}$. A cubic box of about 40 A was used with 2040 molecules. The time step was 2 fs. The long range electrostatics were treated with the Ewald summation with radius of reciprocal summation $\mathrm{k}_{\max }=10$, convergence parameter $\alpha=0.2846$, and a cut off for the real space part of $10 \AA$. These parameters were chosen to minimise the cpu time used and are based on Ewald precision $10^{-5}$. The NPT Nosé-Hoover ensemble was chosen with relaxation times of $0.1 \mathrm{ps}$ for both barostat and thermostat. After equilibration configurations were saved every ps for subsequent analysis. Radial distribution functions and tetrahedral order parameters were calculated on the fly.

All the ionic liquid simulations were carried out at $450 \mathrm{~K}$ using a cubic box of about $23 \AA$ containing 96 ion pairs. The NVT Nosé-Hoover was used with a timestep of 0.5 fs and thermostat relaxation time of $0.1 \mathrm{ps}$. Electrostatics were treated using the Ewald sum with parameters $\mathrm{k}_{\max }=16$ and $\alpha=0.3288$, corresponding to a precision of $10^{-8}$. The long-range cutoff was set to $11 \AA$. Following a short equilibration run, data were collected over a period of $1 \mathrm{~ns}$ with configurations saved every $0.5 \mathrm{ps}$. All analysis was performed post-run on the resulting trajectories using simple Fortran code.

\subsection{Calculation of distributions and order parameters}

Three dimensional probability distributions were calculated by taking each water molecule or cation in the sample in turn, finding its local axis system and forming a histogram of numbers of ring centres or oxygen sites in a cubic box of side $15 \AA$ and grid spacing of $0.5 \AA$.

For water we use the tetrahedral order parameter $Q_{\text {tet }}$ used by Errington and Debenedetti (18), which is based on one introduced by Chau and Hardwick (19). In this measure the local tetrahedral order at each oxygen site $i$ is defined by a sum over the angles $\theta_{j i k}$ subtended by the four nearest neighbours $j, k$ at the 
oxygen site $i$

$$
Q_{i}=1-\sum_{j<k}^{6} \frac{3}{8}\left[\cos \theta_{j i k}+\frac{1}{3}\right]^{2}
$$

The tetrahedral order $Q_{t e t}$ of the liquid is defined as the average of the $Q_{i}$ values at each site. Calculation of this quantity was included in a modified version of DL_POLY and computed at the same time as the radial distribution function (in subroutine rdf0.f).

\section{Case study 1 - Water and modified water models}

Figure 1 shows the OO radial distribution functions for these two families. Considering first the hybrid family, one sees that the first peak moves out and broadens, but the second peak changes in a more interesting way - first disappearing (model s15) and then reappearing (model s30) at a greater distance. This change can be correlated with a loss of tetrahedral structure. An ideal tetrahedral network is characterised by a ratio of 1.63 between nearest neighbours and next nearest neighbours, while in a Lennard-Jones liquid the second peak is at twice the distance of the first peak. The figures in table 2 show that it is only in the $\mathrm{SPC} / \mathrm{E}$ model that this ratio has the value typical of tetrahedral networks; in all other models the ratio is near 2 . This is confirmed by a steady decrease in the tetrahedral order parameter in this family (see Table $2)$.

Figure 2 shows the three dimensional contours of probability functions for finding oxygen sites around a water molecule (20). In the three members of the hybrid family (left hand column - SPC/E, s15 and s30) the most probable positions are along the $\mathrm{OH}$ bonds. In SPC/E water there are two other regions of high water density behind the oxygen, giving an approximate tetrahedral arrangement of neighbouring water molecules in the first solvation shell. At lower probabilities (not shown) one sees a build-up of probability above and below the $\mathrm{OH}$ bonds which Svishchev and Kusalik (20) interpreted as corresponding to a fifth water molecule penetrating the inner shell. In the s15 model the pattern is similar, although the localisation of the neighbouring molecules is less, with rather diffuse regions behind the molecule. Once one reaches the s30 model, the distribution of molecules in the first shell is less well defined. The maxima in the hydrogen bonded positions remain, but there is now a significant probability of finding neighbouring molecules in a ring-shaped region above and below the water molecule. Looking back at the radial distribution functions (figure 1) one sees that these changes are correlated with a broadening and shifting of the position of the first shell.

Figure 3 complements this information by showing the distribution of angles subtended at an oxygen site by pairs of the four nearest neighbours. This is a three-body property and gives some information about the local network structure. In the SPC/E model there is a preference for the tetrahedral angle ( $\cos \theta=-0.33)$, although the distribution is quite broad. There is also a shoulder corresponding to an angle slightly less than $60^{\circ},(\cos \theta=0.5)$, which is probably due to a new molecule entering the first shell in order to displace an existing molecule. In the hybrid models s15 and s30, the tetrahedral peak flattens and a peak at about $\cos \theta=0.45$ grows in as the liquid becomes more Lennard-Jones like. This peak corresponds to a triangular arrangement of molecules. In the most extreme member of this family (s30) where $g(r)$ shows a second shell, one also sees a peak beginning to grow in near $\theta=120^{\circ}(\cos \theta=-0.5)$. Thus in the hybrid family there is decreasing tetrahedral structure in the first shell. In the s30 model, where the hydrogen bond strength has been reduced by about $30 \%$, the tetrahedral structure in the first shell is greatly reduced and does not propagate into the second shell.

The changes seen in the local structure around the bent family of models where the bond angle is reduced are different from that seen in the hybrid family. The first peak in $g(r)$ (see figure 1) broadens in the w90 
model (bond angle 90 ) and then sharpens and moves inwards when the bond angle is reduced to $60^{\circ}$. The second peak behaves in a similar way. This is due to the gradual replacement of the tetrahedral structure by a more linear structure. Figure 2 shows what is happening to the local structure. In model w90 (90 water) the probability distribution in the first shell has a fairly diffuse structure, and is similar to that found in the hybrid model s15 although the two positions corresponding to water molecules accepting hydrogen bonds are at $90^{\circ}$ rather than at $109.5^{\circ}$. In $60^{\circ}$ water there is a qualitative change as there is now only one region of density of molecules accepting hydrogen bonds, and one region for donating molecules. This is because when the bond angle is as small as $60^{\circ}$, a molecule can form a double hydrogen bond to a neighbouring molecule, leading to the formation of chains. The formation of chains can be seen in the bottom right panel of figure 2 which shows the contour surface at $1.5 \mathrm{~g} \mathrm{~cm}^{-3}$ rather than $2 \mathrm{~g} \mathrm{~cm}^{-3}$. In this view (taken down the $\mathrm{HH}$ axis) one can see evidence of chain formation. In the region in the lower part of the figure one sees two regions of enhanced density corresponding to molecules accepting hydrogen bonds in the first shell and second shell molecules in the same chain. This chain formation also accounts for the sharpening of the second peak in $g(r)$ in $60^{\circ}$ water. The three body angular correlations also show this (figure 3 ). In $60^{\circ}$ water there is a maximum in the probability distribution of $\cos \theta$ at $\cos \theta=-1$, corresponding to a linear arrangement of three molecules. There is also a maximum as well as near $\cos \theta=0.5$, corresponding to the bond angle of $60^{\circ}$, showing that there are also triangles of water molecules. The distribution of $\cos \theta$ for $90^{\circ}$ water is nearly uniform except for small angles where the molecules exclude each other. Surprisingly there is no preference for angles of $90^{\circ}(\cos \theta=0)$ in spite of this being the bond angle in the model.

We conclude that in the hybrid family of liquids, where the hydrogen-bond strength is reduced, the local structure becomes less tetrahedral and more close packed. There is a minimum order in the region of the second shell in this family near model s15. This also corresponds to a maximum entropy of the liquid. In the bent family of liquids, on the other hand, the local structure becomes less tetrahedral, but the new structure which replaces it has a tendency to have chains of molecules when the bond angle is decreased sufficiently that double hydrogen bonds can be made between molecules. This local structure is reflected in changes of macroscopic properties of the liquids (7). For example, in the hybrid family the regions of anomalous behaviour become smaller as the intermolecular potential becomes more Lennard-Jones like.

\section{Case study 2 - Hydrogen-bonding interactions in $\left[\mathrm{C}_{1} \mathrm{mim}\right] \mathrm{Cl}$}

Figure 4 shows the effects of modifying the $\mathrm{C}-\mathrm{H}$ bond dipoles of the imidazolium ring while keeping the overall charge on the cation equal to $1 e$. The reference intermolecular potential is the force-matched forcefield of Reference (17), which gives anion probability functions which are in good agreement with $a b$ initio results. In the figure two views of the probability density of anions and cations are given for each model. Even for models with a small change in the $\mathrm{C}-\mathrm{H}$ dipole (small $\delta$ ) where the positive character of the hydrogen atoms is still retained, there are notable changes in the anion density distribution. In particular, the region of high anion density directly along the unique $\mathrm{C}-\mathrm{H}$ bond splits into two, each moving out to a position between the $\mathrm{C}-\mathrm{H}$ group and the methyl groups. Similarly, the lobes associated with the hydrogens at the bottom of the ring move upwards towards the methyl groups. As long as the dipole is directed outwards along the $\mathrm{C}-\mathrm{H}$ bonds $(\delta<0.5)$ there is a region of high cation density above and below the plane of the ring. However once the dipoles are reversed, at $\delta=0.6$ this region has disappeared and new lobes have appeared in the region along the N-Me vector.

Approaching the complete reversal of the bond dipole $(\delta=0.8$ and $\delta=1.0)$ we tend towards a model that has had its three main hydrogen bonding sites removed, and replaced with new sites whose atomic charges actively repel chloride anions. The original regions of high anion density anion have shifted such that the main interaction between cation and chloride is through weak association with the methyl groups, in themselves poor hydrogen bond donors. More striking is the replacement of cation density above and below the plane of the ring with anion density. Of course, the charges in the reversed $\mathrm{C}-\mathrm{H}$ dipole model 


\section{Conclusions}

The changes that take place are well-illustrated in the ion-ion radial distribution functions, figure 6 , calculated between the centres-of-mass of the ions. The single peak in the cation-anion curve at $4.5 \mathrm{~A}$ in the unmodified model decreases as the $\mathrm{C}-\mathrm{H}$ dipoles are reversed, splitting into two separate peaks one at $3.2 \AA$ associated with the anions above and below the ring, and a second smaller peak at 5.1 $\AA$ associated with anions interacting weakly with the methyl groups. As observed in the $3 \mathrm{~d}$ probability distributions, though, the structural differences reflected through these radial distribution functions are minimal for small $\delta$. Cation-cation structuring is weak in the unmodified potential, resulting in a single broad peak in the radial distribution function. Reversing the $\mathrm{C}-\mathrm{H}$ dipoles, however, promotes an increase in this structuring with a well-defined (if slightly flattened) peak appearing at $6 \mathrm{~A}$.

A plot of the same three dimensional probability distributions at a lower contour surface (figure 5) reveals how, in the model where $\delta=1.0$, the positions of higher density in the cation distribution are remarkably similar to the positions of the anion lobes in the original model. Instead of a distinct primary solvation shell of anions followed by a secondary shell of cations, in the reversed dipole model the cations visibly begin to encroach into the primary shell. Average numbers of hydrogen bonds from individual cations reflect this trend - for the unmodified potential the average is 6.2 compared to 3.6 when $\delta=1.0$, and defining a hydrogen bond as when $r_{\mathrm{C}-\mathrm{Cl}}<4.0$ and $\widehat{\mathrm{CHCl}}>120.0$. The number of interactions with methyl hydrogens remains the same in both models, each individual hydrogen involved in $0.6 \mathrm{H}$-bonds on average.

Small perturbations of the $\mathrm{C}-\mathrm{H}$ bond dipoles (viz. charges) in the model do not promote significant change in the structural features in the system, suggesting that there exists a 'window' of atomic charge sets that will give the correct liquid structure, but which may give widely varying energetic and thermophysical properties. Indeed, as seen in Reference (17), a forcefield that reproduces well the structure of the liquid can display some error in the bulk density. However the details of the distributions from many classical force fields such as the original one of Hanke et al. (15) give anion distributions which are somewhat more similar to the model with $\delta=0.2$ than to the reference model (which gives good agreement with the $a b$ initio calculations). Beyond a certain threshold, though, the liquid structure becomes extremely sensitive to the local electrostatic potential of the cation, so much so that cations that were previously to be found above and below the plane of the imidazolium ring are replaced with anions owing to the highly disfavoured interaction now associated with the ring hydrogens.

Since a modest amount of effort must be expended in order to alter the ion structuring in the liquid, this indicates that there is a significant amount of hydrogen bonding in the liquid and that it is a dominant influence over the ionic structuring in these systems.

These two case studies show the power of classical molecular dynamics simulation to investigate the dependence of local molecular structure on details of the intermolecular potentials. In water we have shown how the local structure responds to either changes in hydrogen bond strength or changes in the bond angle. In further studies we have looked at the consequent changes in anomalous properties (7) and we are currently examining solvation properties of the modified water models to see the connection between local structure and solvation. In the second case study we have showed the response of the local 
structure to the hydrogen bond strength of the ring hydrogens in the cation. This approach is also being applied to the charges of the ions, and dynamical effects of both modifications will be assessed.

Both studies have used the DL_POLY suite of programs and subroutines with modifications that we have made to suit particular problems or to suit our prejudices concerning output files and their format. The availability of the source code is a strength for the professional user.

\section{Acknowledgements}

We thank J. Kohanoff, M. Del Pópolo and T. Head-Gordon for discussions and EPSRC (grants GR/S41562 and EP/D029538/1) and the Leverhulme Trust (Emeritus Fellowship to RMLB) for financial support. 


\section{References}

[1] N. Metropolis, A.W. Rosenbluth, M.N. Rosenbluth, A.H. Teller, E. Teller. Equation of state calculations by fast computing machines. J. Chem. Phys 21, 1087 (1953).

[2] A. Rahman. Correlations in the motions of atoms in liquid argon. Phys. Rev. A, 136, 405 (1964).

[3] G.D. Harp, B.J. Berne. Linear and angular momentum autocorrelation functions in diatomic liquids. J. Chem. Phys., 49, 1249 (1968).

[4] M.P. Allen, D.J. Tildesley. Computer Simulation of Liquids. Oxford University Press (1987).

[5] W. Smith, T.R. Forester. The DL_POLY manual, Daresbury Laboratory. http://www.cse.clrc.ac.uk/msi/software/DL_POLY (1996).

[6] D.L. Bergman, R.M. Lynden-Bell. Is the hydrophobic effect unique to water? The relation between solvation properties and network structure in water and modified water models. Mol. Phys., 99, 1011 (2001).

[7] R.M. Lynden-Bell, P.G. Debenedetti. Computational investigation of order, structure, and dynamics in modified water models. J. Phys. Chem. B, 109, 6527 (2005).

[8] P.A. Hunt. The simulation of imidazolium-based ionic liquids. Mol. Sim., 32, 1(2006).

[9] C. Hardacre, J.D. Holbrey, S.E.J. McMath, D.T. Bowron, A.K. Soper. Structure of molten 1,3dimethylimidazolium chloride using neutron diffraction. J. Chem. Phys., 118, 273 (2003).

[10] M.G. Del Pópolo, R.M. Lynden-Bell, J. Kohanoff. Ab initio molecular dynamics simulation of a room temperature ionic liquid. J. Phys. Chem. B, 109, 5895 (2005).

[11] M. Bühl, A. Chaumont, R. Schurhammer, G. Wipff. Ab initio molecular dynamics of liquid 1,3dimethylimidazolium chloride. J. Phys. Chem. B, 109, 18591 (2005).

[12] B.L. Bhargava, S. Balasubramanian. Intermolecular structure and dynamics in an ionic liquid: A CarParrinello molecular dynamics simulation study of 1,3-dimethylimidazolium chloride. Chem. Phys. Lett., 417, 486 (2006).

[13] N.E. Heimer, R.E. Del Sesto, Z. Meng, J.S. Wilkes, W.R. Carper. Vibrational spectra of imidazolium tetrafluoroborate ionic liquids. J. Mol. Liquids, 124, 84 (2006).

[14] R.W. Berg, M. Deetlefs, K.R. Seddon, I. Shim, J.M. Thompson. Raman and ab initio studies of simple and binary 1-alkyl-3-methylimidazolium ionic liquids. J. Phys. Chem. B, 109, 19018 (2005).

[15] C.G. Hanke, S.L. Price, R.M. Lynden-Bell. Intermolecular potentials for simulations of liquid imidazolium salts. Mol. Phys., 99, 801 (2001).

[16] H.J.C. Berendsen, R.J. Grigera, T.P. Straatsma. The missing term in effective pair potentials. J. Phys. Chem., 91, 6269 (1987).

[17] T.G.A. Youngs, M.G. Del Pópolo, J. Kohanoff. Development of complex classical forcefields through force matching to ab initio data: application to a room-temperature ionic liquid. J. Phys. Chem. B, 110, 5697 (2006).

[18] J.R. Errington, P.G. Debenedetti. Relationship between structural order and the anomalies of liquid water. Nature, 409, 318 (2001).

[19] P.-L. Chau, A.J. Hardwick. A new order parameter for tetrahedral configurations. Mol. Phys., 93, 511 (1998).

[20] I.M. Svishchev, P.G. Kusalik. Structure in liquid water - a study of spatial-distribution functions. J. Chem. Phys., 99, 3049 (1993).

[21] A. K. Soper. Empirical potential Monte Carlo simulation of fluid structure. Chem. Phys., 202, 295 (1996). 
Table 1. Potential parameters for modified water models.

2

3

4

5

6

7

8

9

10

11

12

13

14

15

16

17

18

19

20

21

22

23

24

25

26

27

28

29

30

31

32

33

34

35

36

37

38

39

40

41

42

43

44

45

46

47

48

49

50

51

52

53

54

55

56

57

58

59

60

\begin{tabular}{r|ccccc}
\hline model & $q_{O} / \mathrm{e}$ & $\epsilon_{O O} / \mathrm{kJ}$ & $\sigma_{O O} / \AA$ & $\widehat{H O H}$ & $r_{O H}$ \\
\hline SPC/E & -0.8479 & 0.6502 & 3.165 & $109.5^{\circ}$ & 1.0 \\
hybrid: s15 & -0.8479 & 0.9753 & 3.165 & $109.5^{\circ}$ & 1.0 \\
hybrid: s30 & -0.8479 & 1.9505 & 3.165 & $109.5^{\circ}$ & 1.0 \\
bent: w90 & -0.8479 & 0.6502 & $3.165,3.05$ & $90.0^{\circ}$ & 0.816 \\
bent: w60 & -0.8479 & 0.6502 & 2.92 & $60.0^{\circ}$ & 0.667 \\
\hline
\end{tabular}

Table 2. Liquid properties of modified water models.

\begin{tabular}{r|ccccc}
\hline model & $Q_{\text {tet }}$ & PE in liquid / $\mathrm{kJ} \mathrm{mol}^{-1}$ & $\begin{array}{c}\text { first peak } \\
\text { position / A }\end{array}$ & $\begin{array}{c}\text { ratio 2nd:1st } \\
\text { peak position }\end{array}$ & $\begin{array}{c}\text { relative dimer } \\
\text { H-bond strength }\end{array}$ \\
\hline SPC/E & 0.63 & -46.3 & 2.74 & 1.6 & $100 \%$ \\
hybrid: s15 & 0.56 & -41.6 & 2.90 & - & $87 \%$ \\
hybrid: s30 & 0.48 & -41.7 & 2.93 & 2.0 & $69 \%$ \\
bent: w90 & 0.45 & -33.6 & 2.78 & 1.9 & 1.9 \\
bent: w60 & 0.39 & -35.9 & 2.60 & & \\
\hline
\end{tabular}


2

3

4

5

6

7

8

9

10

11

12

13

14

15

16

17

18

19

20

21

22

23

24

25

26

27

28

29

30

31

32

33

34

35

36

37

38

39

40

41

42

43

44

45

46

47

48

49

50

51

52

53

54

55

56

57

58

59

60
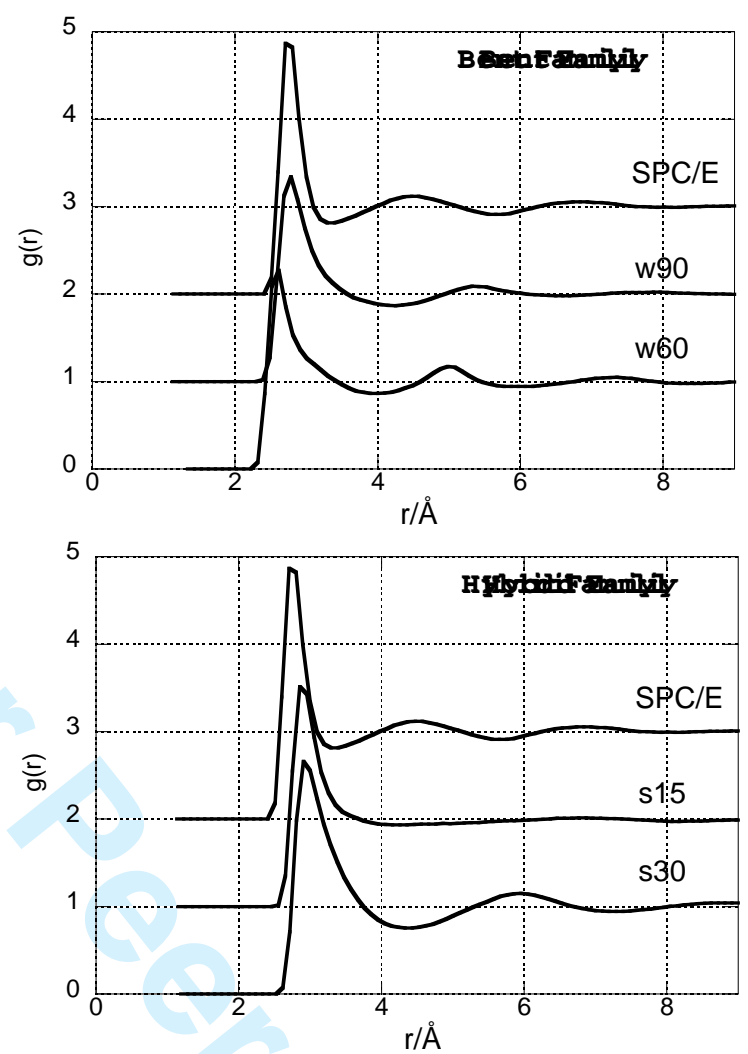

Figure 1. OO Radial distribution functions for the bent family of models (above) and the hybrid family (below). In the bent family the peaks move out, but sharpen at $60^{\circ}$, while in the hybrid family the second peak first disappears and then reappears further out. 
a)

SPC/E

b)
515

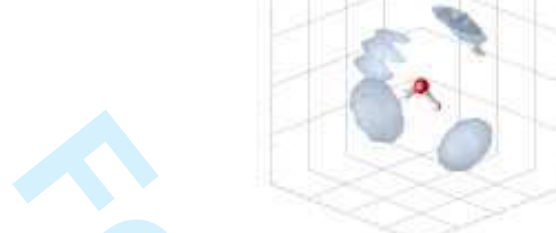

c)

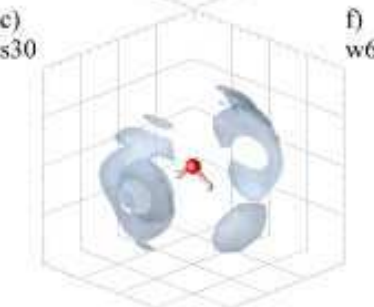

d)

w90

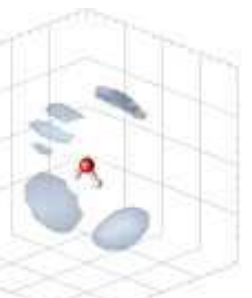

e)

w 60

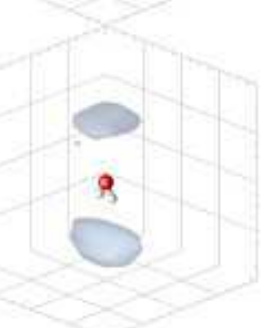

f)

w60

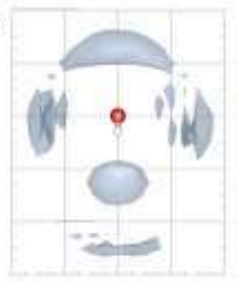

Figure 2. Views of three dimensional contours of the density distribution of molecules around a water for the two families of models. Left - from top to bottom: (a) SPC/E; (b) hybrid model s15; (c) hybrid model s30. Right - from top to bottom: (d) bent model w90 $\left(90^{\circ}\right.$ water); (e) and (f) bent model w60 (60 ${ }^{\circ}$ water). With the exception of (f) contours are drawn at twice the average density; (f) shows contours at 1.5 times the average density. Grid interval shown is $2 \mathrm{~A}$. 
2

3

4

5

6

7

8

9

10

11

12

13

14

15

16

17

18

19

20

21

22

23

24

25

26

27

28

29

30

31

32

33

34

35

36

37

38

39

40

41

42

43

44

45

46

47

48

49

50

51

52

53

54

55

56

57

58

59

60
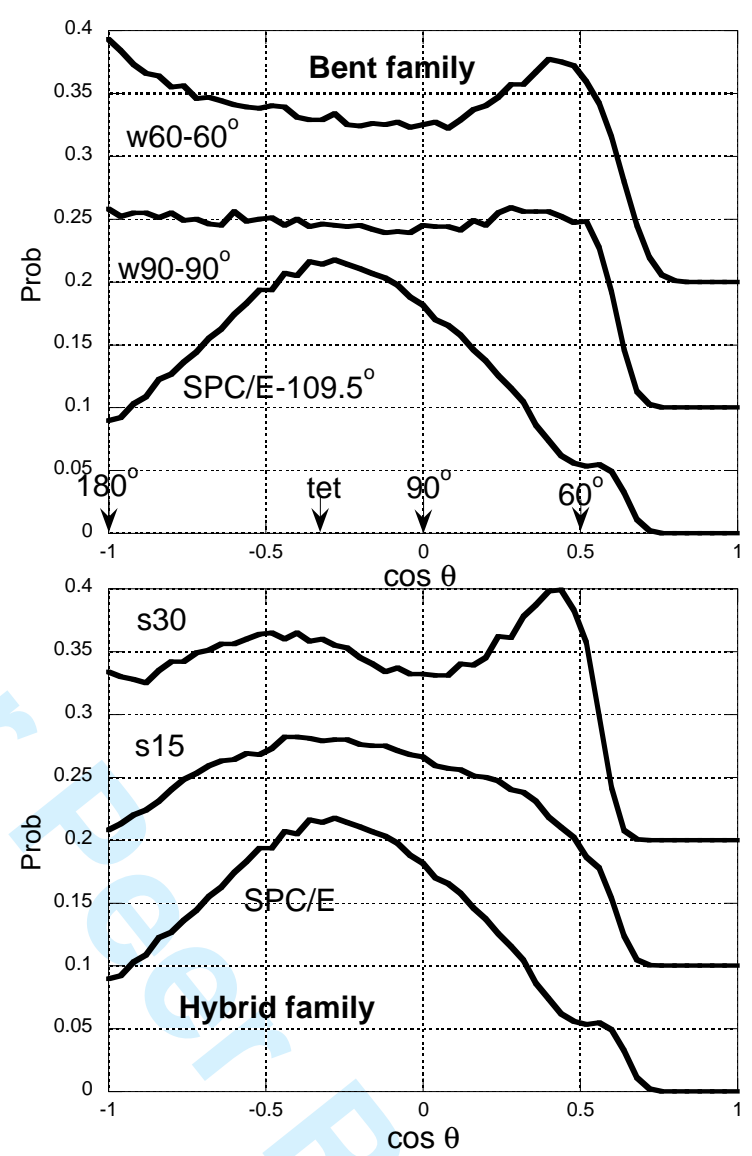

Figure 3. Distribution of cosines of angles subtended by near neighbours for the bent family (above) and the hybrid family (below). A random arrangement of molecules would give a flat probability distribution. The preferred arrangement changes from near tetrahedral $(\mathrm{SPC} / \mathrm{E})$ through random to a preference for linear and triangular $\left(60^{\circ}\right)$ patterns in the bent family, and to triangular for the hybrid family. 

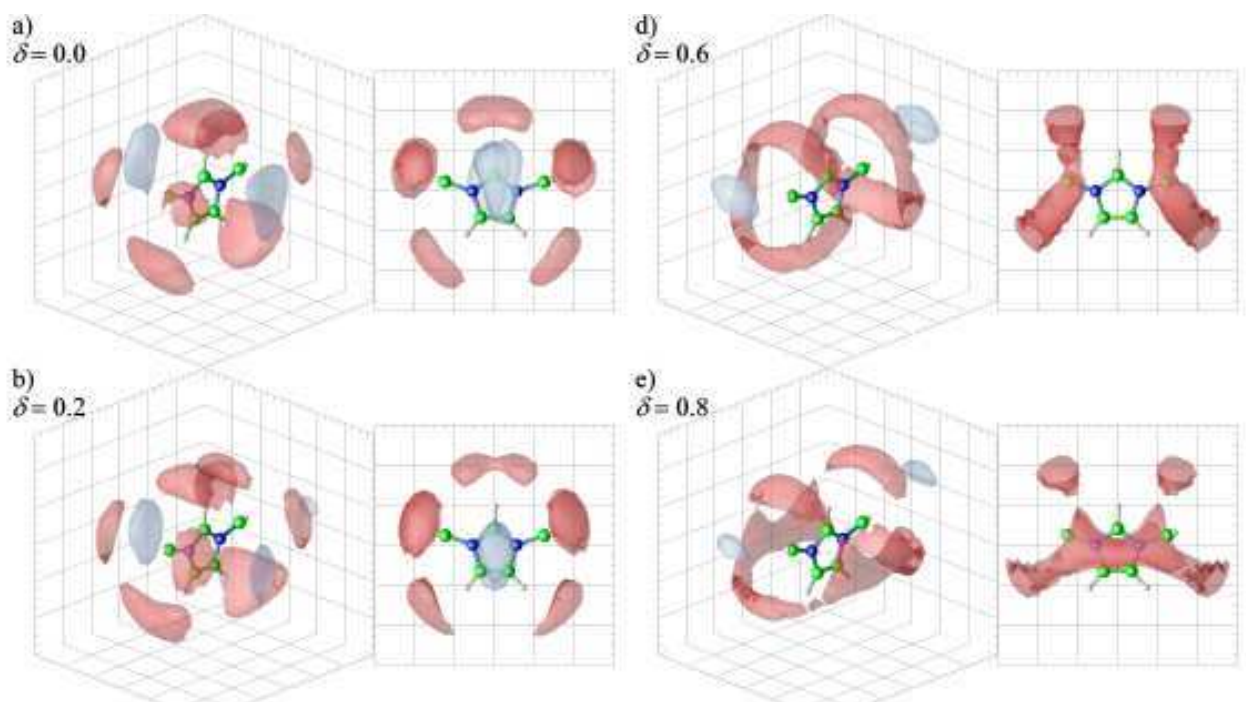

e) $\delta=0.8$
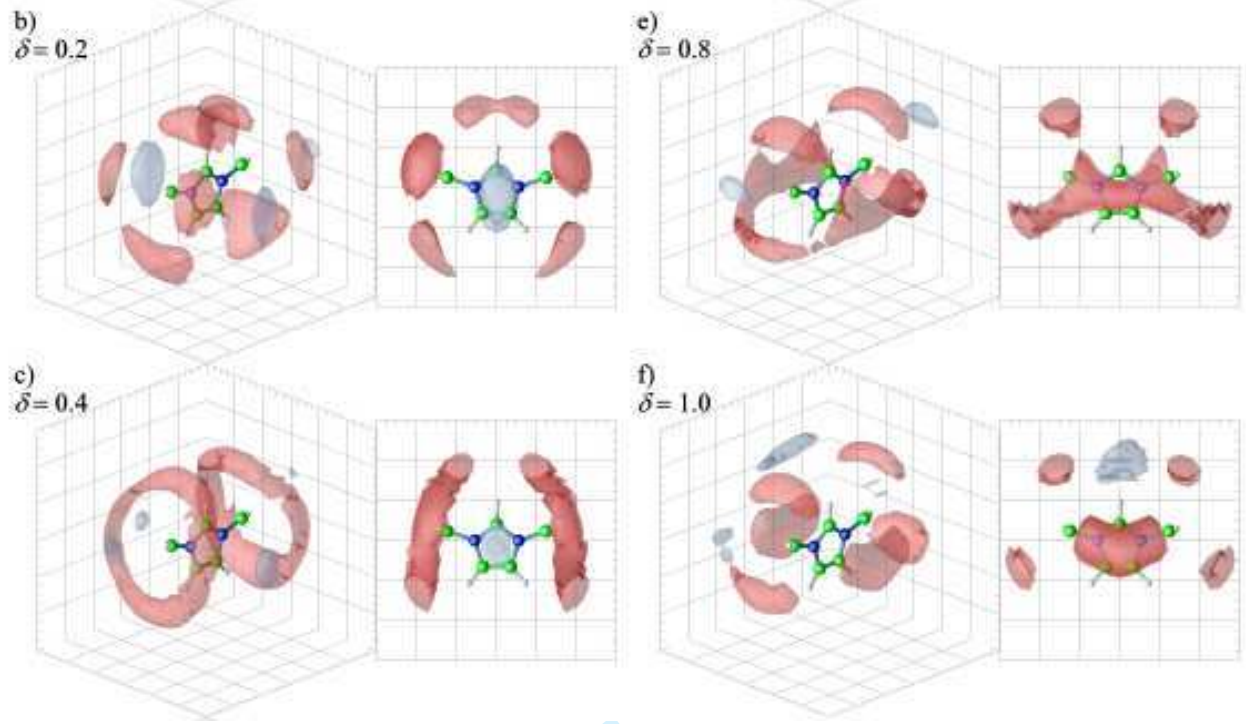

f) $\delta=1.0$

Views of the three dimensional contour surfaces of the density distribution of [C $\left.\mathrm{C}_{1} \mathrm{mim}\right]$ cations (blue) and chloride anions (red) about cations. Two views are given for each model. Results from the unmodified potential are shown in (a), while (b) - (f) show the results of gradual reversal of the imidazolium $\mathrm{C}-\mathrm{H}$ bond dipoles. Anion contours are drawn at 5 times the average density, while cation contours are drawn at three times the average. Grid interval shown is $2 \mathrm{~A}$. 


\section{Page 15 of 22}

1

2

3

4

5

6

7

8

9

10

11

12

13

14

15

16

17

18

19

20

21

22

23

24

25

26

27

28

29

30

31

32

33

34

35

36

37

38

39

40

41

42

43

44

45

46

47

48

49

50

51

52

53

54

55

56

57

58

59

60
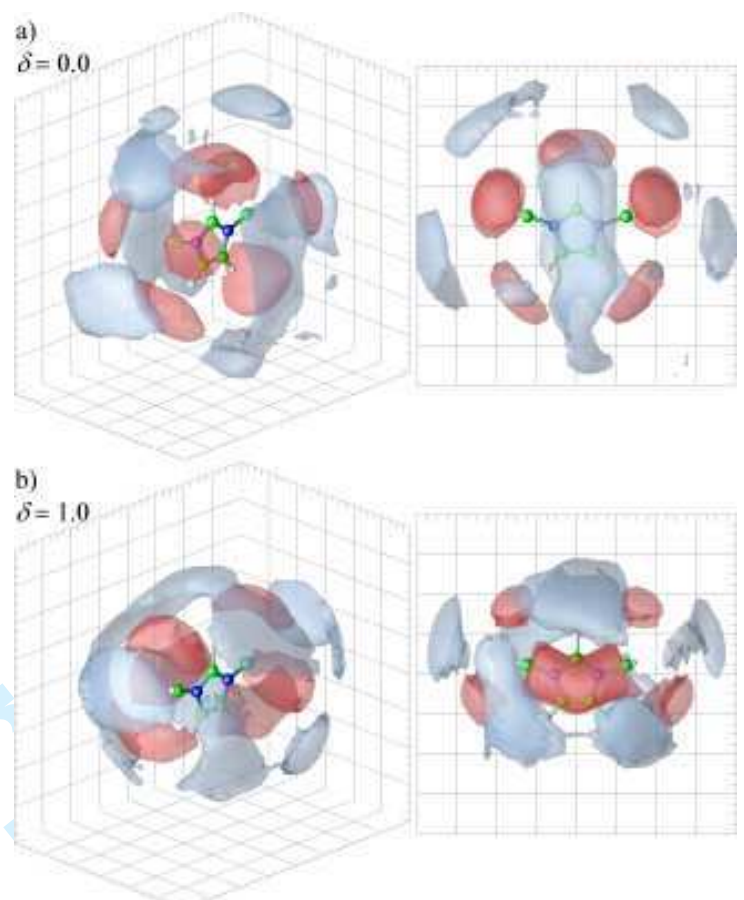

Figure 5. Views of the three dimensional contours of the density distributions of $\left[\mathrm{C}_{1}\right.$ mim] cations (blue) and chloride anions (red) about cations for the unmodified potential (a) and for the model with the reversed $\mathrm{C}-\mathrm{H}$ dipoles (b). Two views are given for each model. Contours are drawn at twice and four times the average for cations and anions respectively. Grid interval shown is $2 \mathrm{~A}^{\circ}$. 

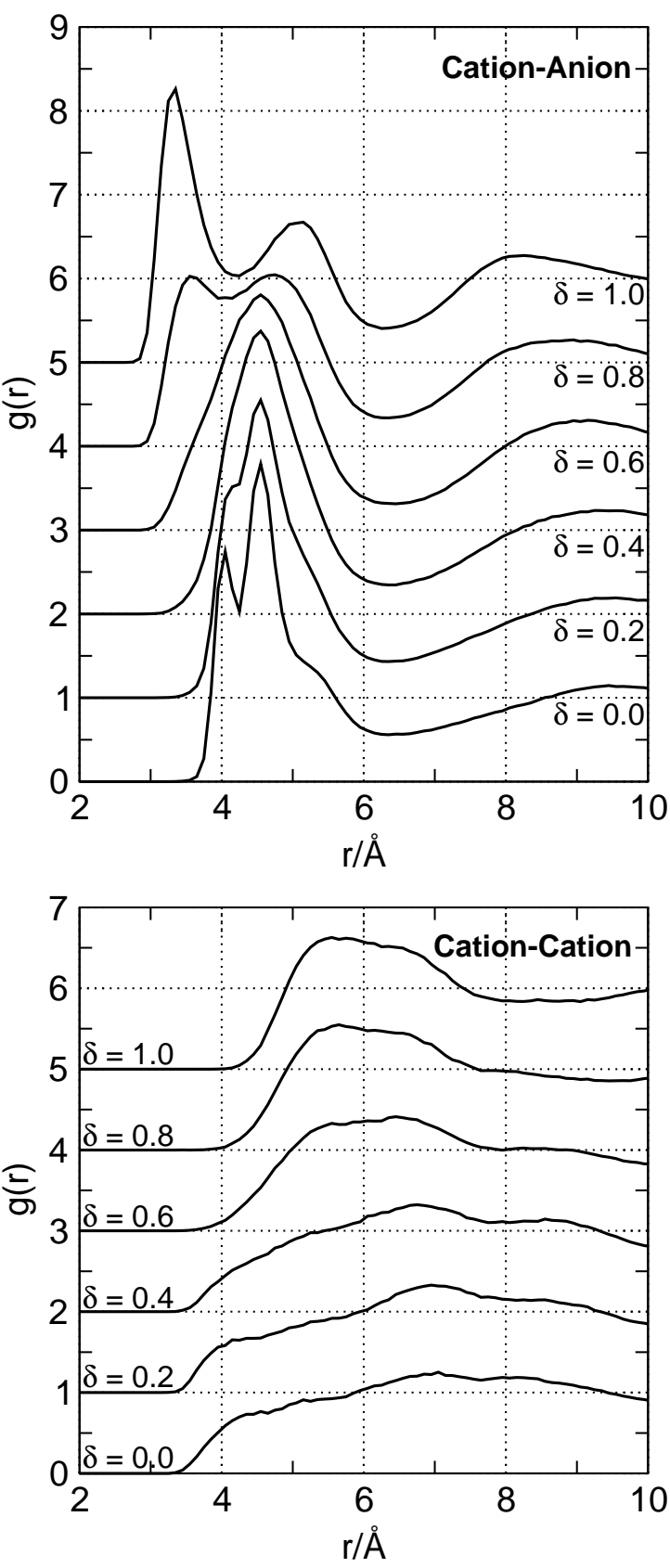

Figure 6. Radial distribution functions of cations and anions about cations in the ionic liquid $\left[\mathrm{C}_{1} \operatorname{mim}\right] \mathrm{Cl}$. Reversing the $\mathrm{C}-\mathrm{H}$ dipoles has the effect of splitting the cation-anion peak in two (a), and also promotes a slightly stronger structuring of cations (b). 

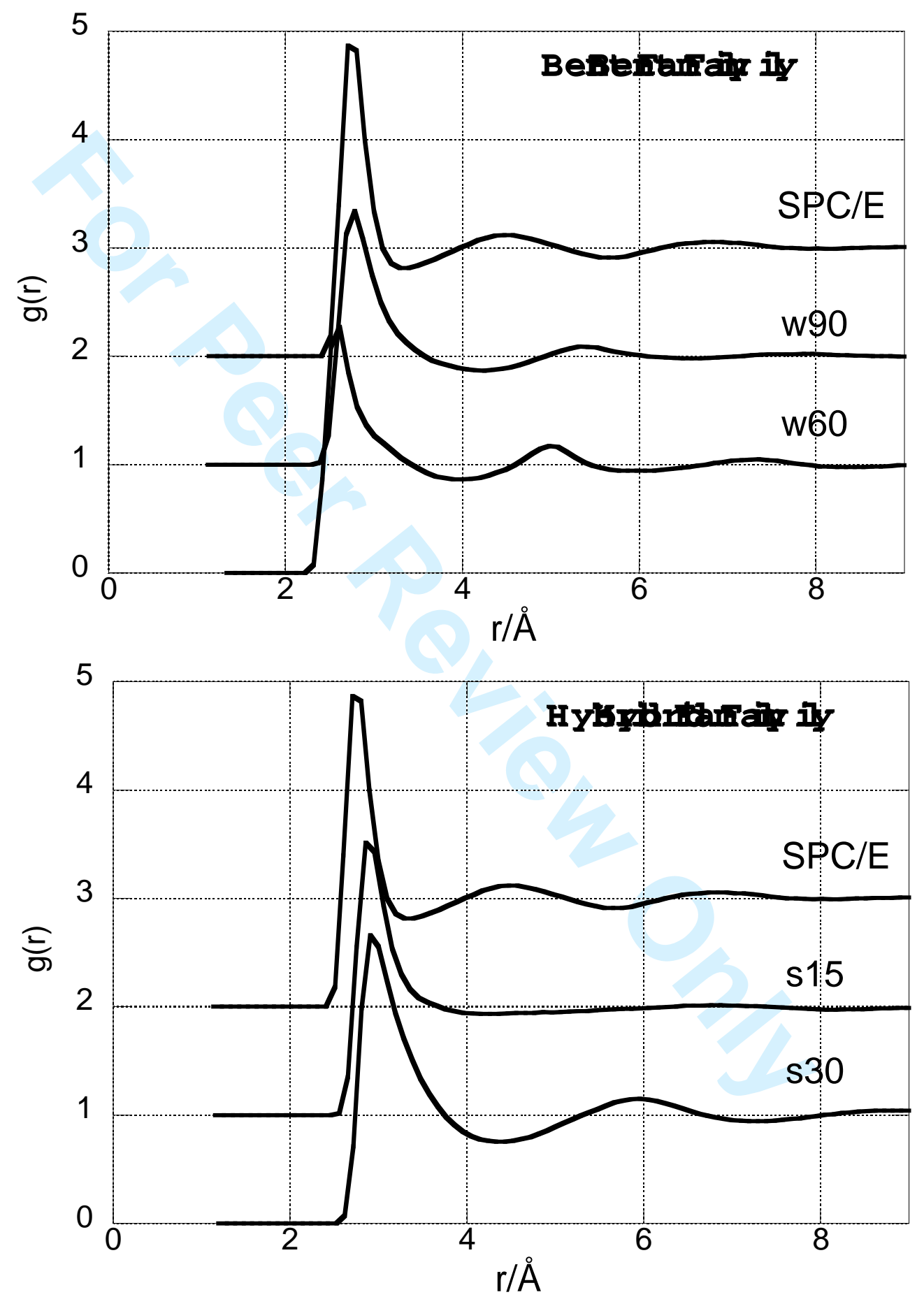

http://mc.manuscriptcentral.com/tandf/jenmol 
a)

b)

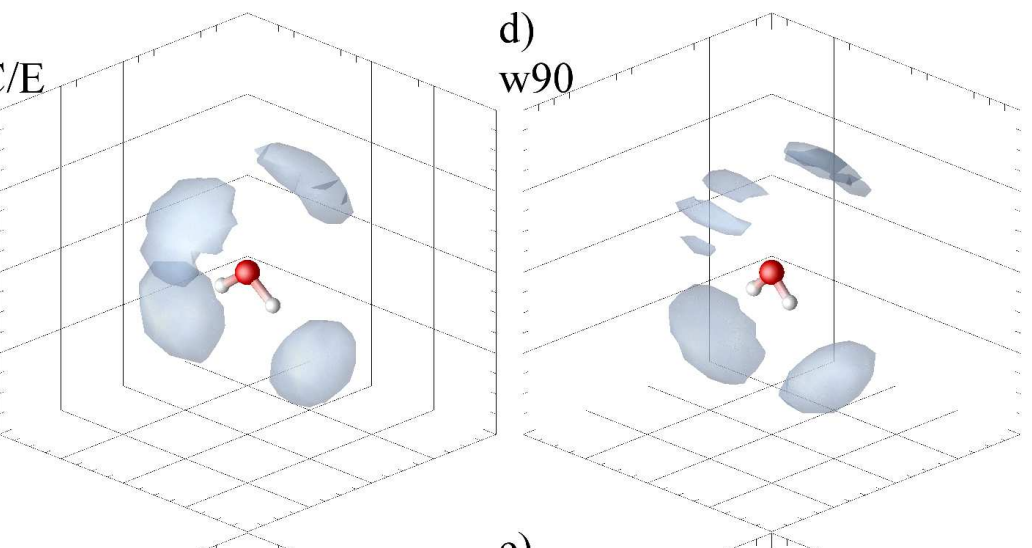

s15

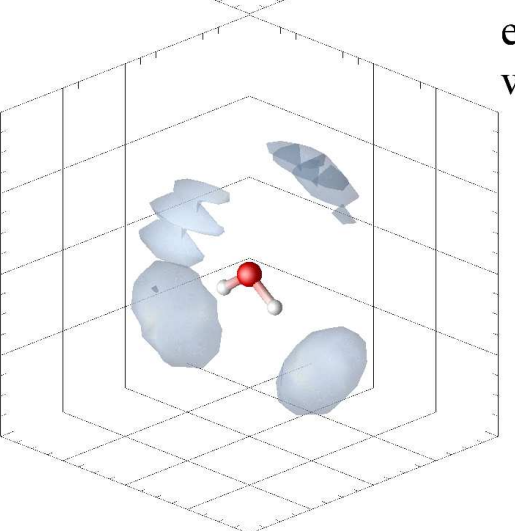

e)

c)

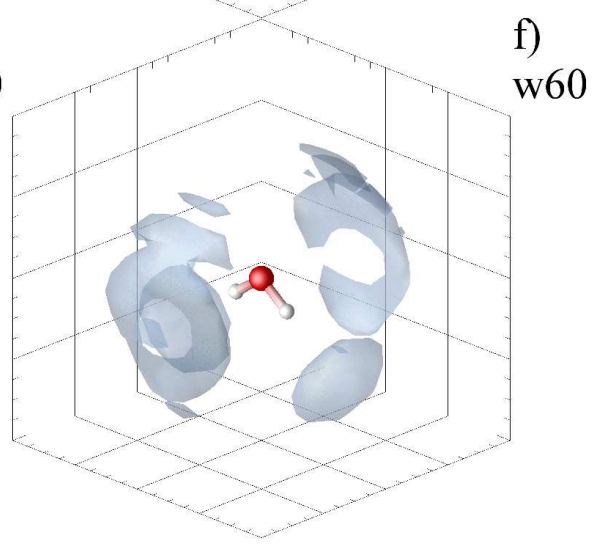

f) w60

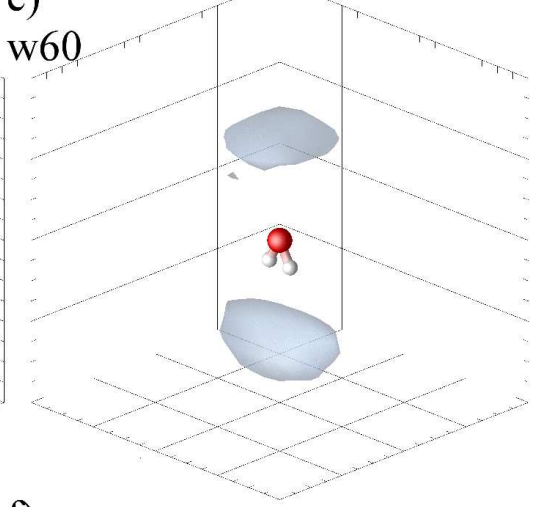

w60

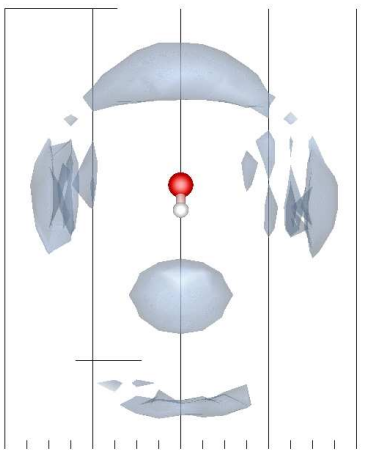

Views of three dimensional contours of the density distribution of molecules around a water for the two families of models.

Left - from top to bottom: (a) SPC/E; (b) hybrid model s15; (c) hybrid model s30.

Right - from top to bottom: (d) bent model w90 ( $90^{\circ}$ water); (e) and (f) bent model w60 ( $60^{\circ}$ water). With the exception of (f) contours are drawn at twice the average density; (f) shows contours at 1.5 times the average density. Grid interval shown is $2 \AA$. $1148 \times 1503 \mathrm{~mm}(72 \times 72 \mathrm{DPI})$ 

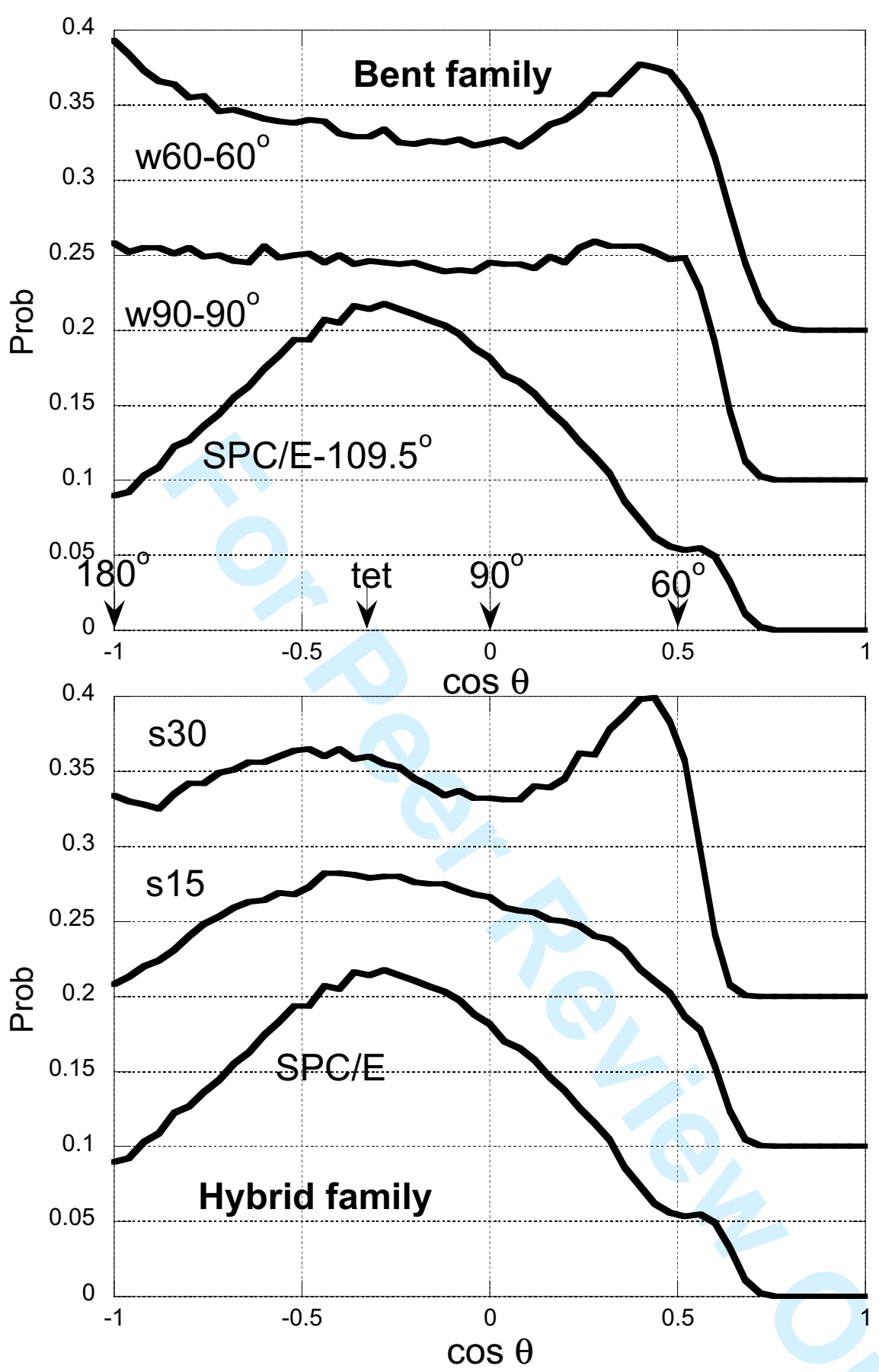

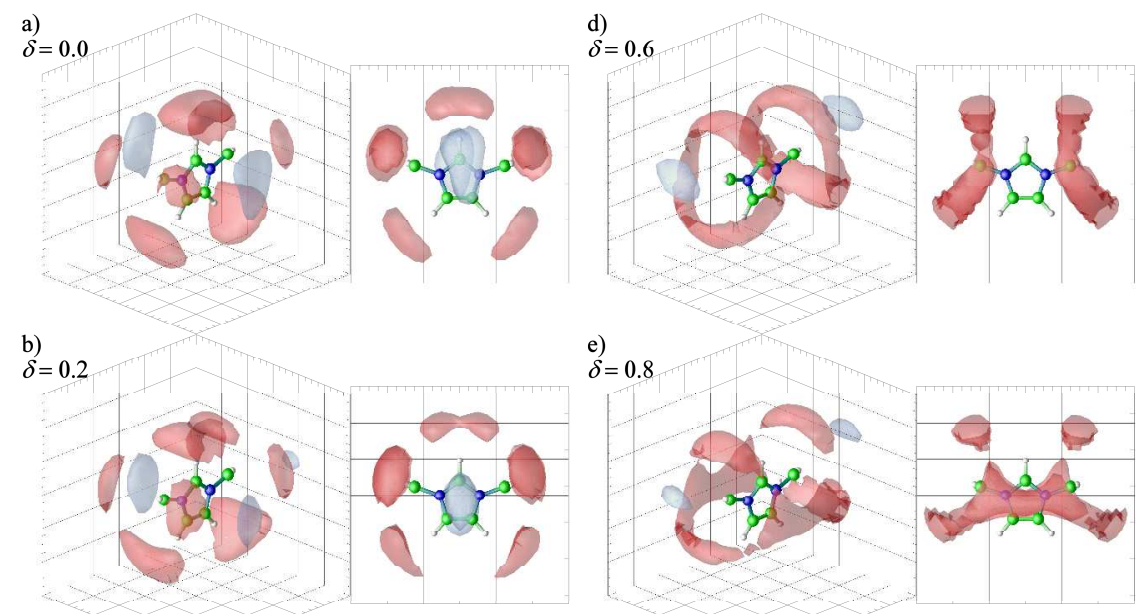

$\stackrel{\text { e) }}{\delta=0.8}$

$\stackrel{c}{8)}=0.4$

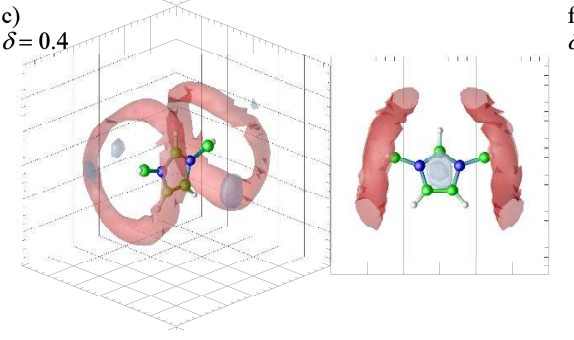

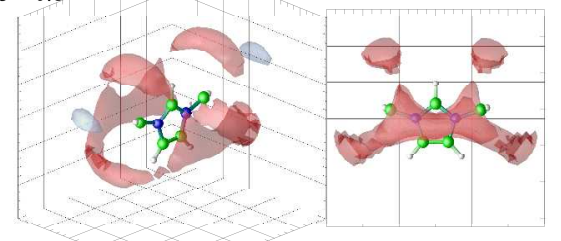
${ }_{\delta=1.0}$

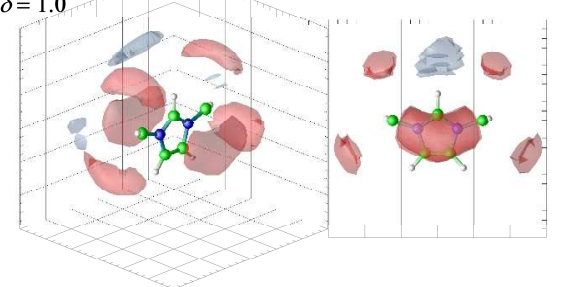

Views of the three dimensional contour surfaces of the density distribution

of [ $\left.\mathrm{C}_{1} \mathrm{mim}\right]$ cations (blue) and chloride anions (red) about cations. Two views are

given for each model. Results from the unmodified potential are shown in (a), while (b) - (f) show the results of gradual reversal of the imidazolium $\mathrm{C}-\mathrm{-H}$ bond dipoles. Anion contours are drawn at 5 times the average density, while cation contours are drawn at three times the average. Grid interval shown is $2 \AA$. $2282 \times 1693 \mathrm{~mm}(72 \times 72 \mathrm{DPI})$ 


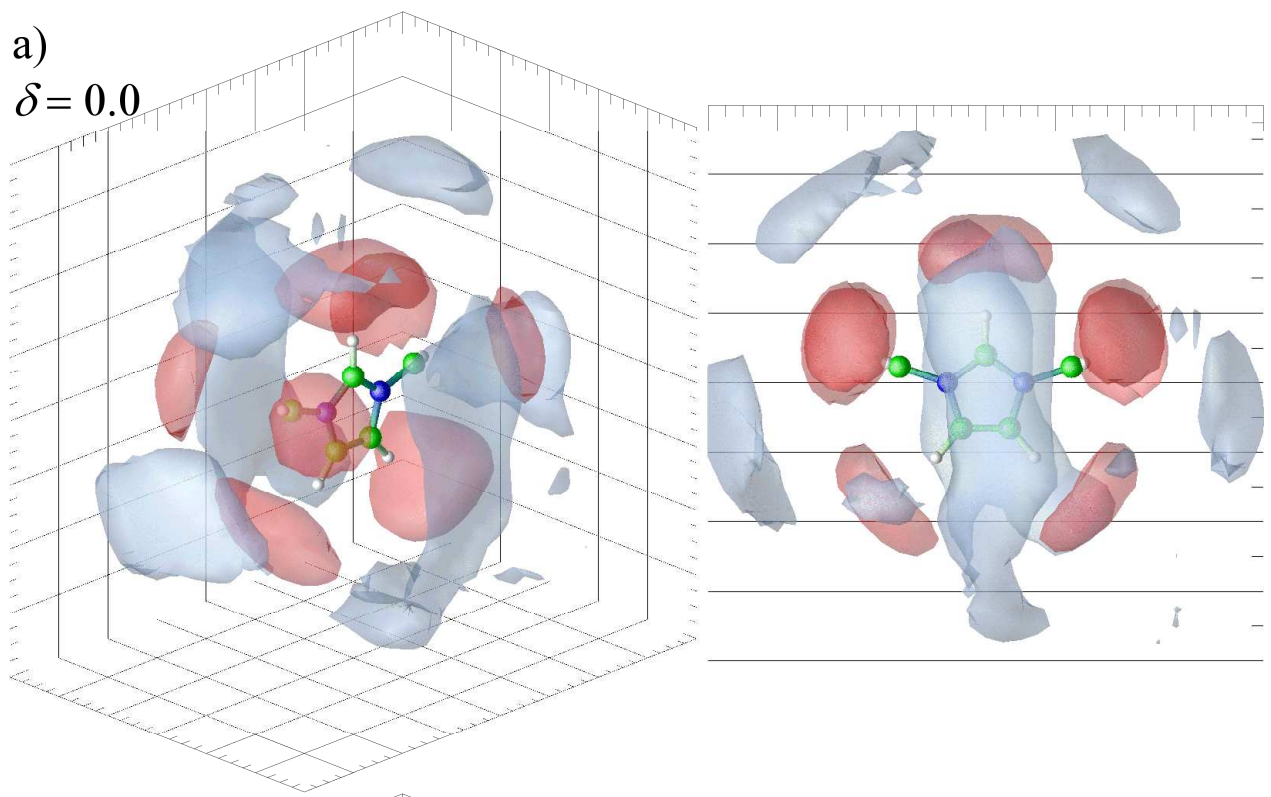

b)

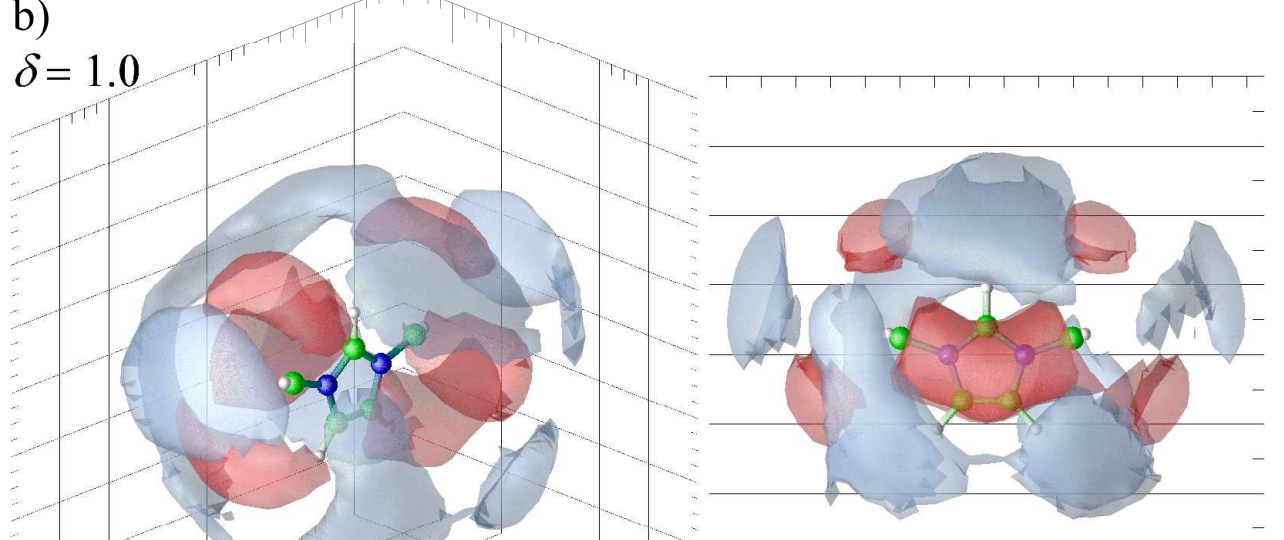

Views of the three dimensional contours of the density distributions of [ $\left.\mathrm{C}_{1} \mathrm{mim}\right]$ cations (blue) and chloride anions (red) about cations for the unmodified potential (a)

and for the model with the reversed $\mathrm{C}-\mathrm{H}$ dipoles (b). Two views are given for each model. Contours are drawn at twice and four times the average for cations and anions respectively. Grid interval shown is $2 \AA$. $1148 \times 1429 \mathrm{~mm}(72 \times 72 \mathrm{DPI})$ 

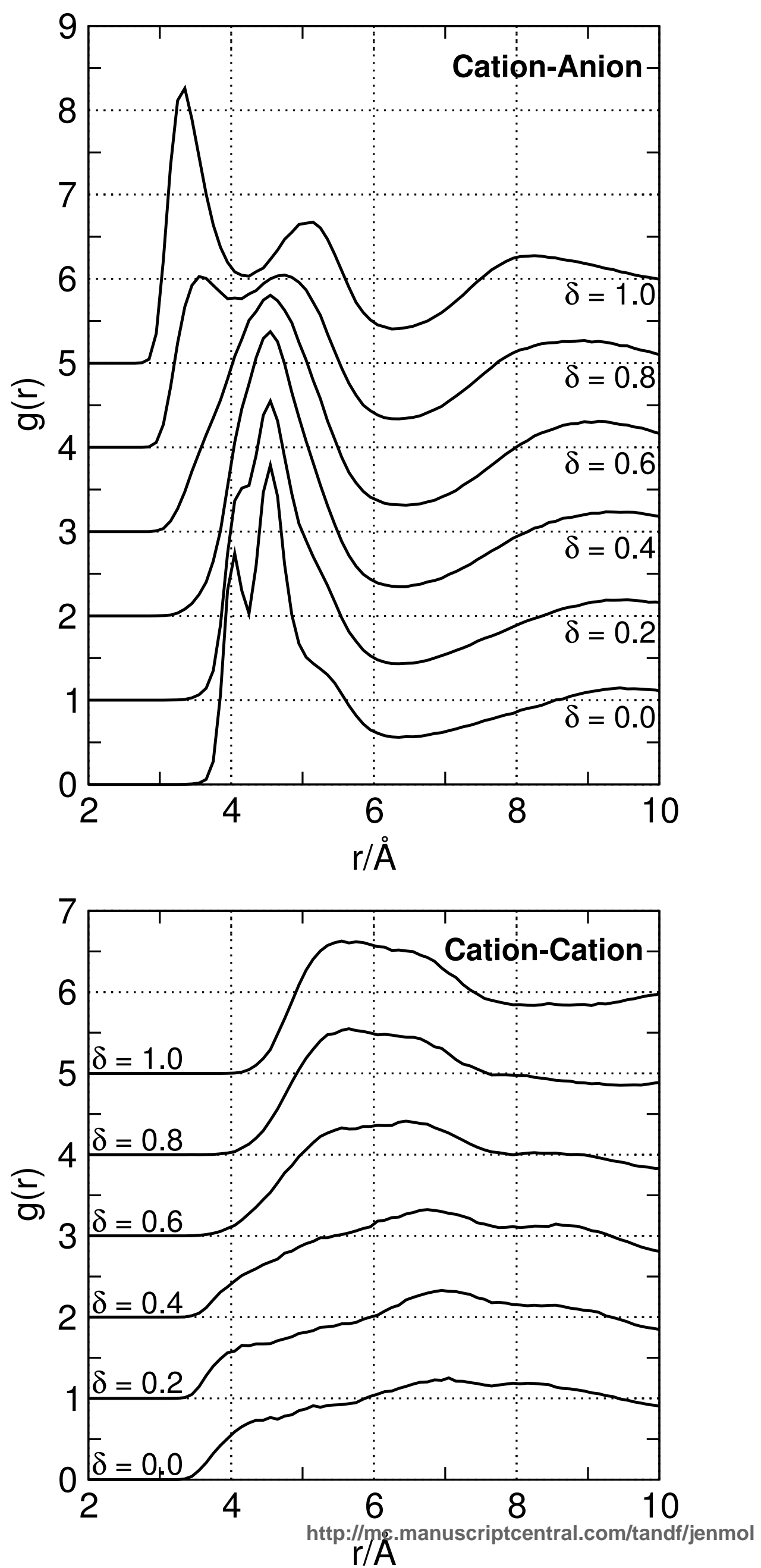